\title{
Driver's Doziness Revelation and Alert System
}

\author{
Miss Prachi Patil ${ }^{1}$, Mr Sunny Patil ${ }^{1}$, Mr. Bipin Pawshe ${ }^{1}$, Prof. Ankit Sanghavi ${ }^{2}$ \\ UG Scholar, Dept. of Computer Engg, Alamuri Ratnamala Institute of Engineering and Technology, Mumbai, India ${ }^{1}$ \\ Asst. Professor, Dept. of Computer Eng, Alamuri Ratnamala Institute of Engineering and Technology, Mumbai, India ${ }^{2}$
}

\begin{abstract}
Driver's invigilance is an important cause for most mishap related to the vehicles crashes. Drivers fatigue resulting from sleep impoverishment or sleep disorders is an important factor in the increasing number of the mishap on today's roads. Dozy driver alert system can form the basis of the system to possibly decrease the mishap related to driver's doziness. The aim of such a system is to accomplish revelation of driver fatigue by placing the camera inside the car, we can keep track of the face of the driver and look for the eye-motion which point out that the driver is no longer in condition to drive. In such a case, a alert signal should be issued. We also illustrate a method that can incline if the eyes are open or closed. The main principle of this system is that it must be highly non-invasive and it should start easily when the key is put in ignition and engine starts and car should run smoothly, without having at the driver begin the system. A dozy driver alert system using image processing as well as accelerometer is propounded in this paper.
\end{abstract}

Keywords: Dozy Driver Alert System, Image Processing, Driver’s invigilance, Vehicles Crashes.

\section{INTRODUCTION}

The purpose of this paper is to develop and describe a prototype of drowsy driver warning system. Our whole focus and concentration will be placed on designing the system that will accurately monitor the open and closed state of the driver's eye in real time.

By continually monitoring the eyes, it can be seen that the symptoms of driver fatigue can be detected early enough to avoid an mishaps. This revelation can be done using a sequence of images of eyes as well as face and head movement.

The examination of eye movements and its edges for the revelation will be used. Devices to revel when drivers are falling asleep and to provide warnings to alert them of the risk, or even control the vehicle's movement, have been the subject to much research and development. Driver fatigue is a critical problem resulting in many thousands of road mishaps each year.

It is not currently possible to calculate the exact number of sleep related mishaps because of the difficulties in reveling whether fatigue was a factor

and in assessing the level of fatigue. However research conveys that up to $25 \%$ of mishaps on monotonous roads in India are fatigue related. Research in other countries also specifies that driver fatigue is a critical problem.

\section{LITERATURE SURVEY}

Driver's languor is among the most common reason for lethal road mishap all over world. This shows that in the transportation industry especially, where a driver of a heavy vehicle is often evoked to hours of continuous driving which causes languor without common leisure period.
Due to the frequent occurrence of driver fatigue this has become an area of great socio-economic concern. For many years revelation of driver's doziness is been done using several techniques.

The work done takes advantage of some maw geometrical lineaments to detect carvenous. The work propounds the revelation and distinction between the two pictures of face. Revelation of driver's cavernous is done based on the distance between chin and nostrils. Uses Gravity-centre template to detect the face. Gabor wavelets and grey projection is been used for revelation of the maw nook.

Finally LDA is applied to classify feature vectors to detect yawning. It represents a technique where face is situated through an approach used for video framing of ViolaJones face revelation. From the face area, a maw window is evoked; wherein brim is been examined through specialFlossy C Means clustering. In there is an advantage of two cameras: a low resolution camera for the face and a high resolution one for the maw [1]. It then uses haar-like features to detect driver's mouth and yawning is detected by the ratio of mouth height and width [2]. A technique is espoused for carvenous revelation based on the changes in maw geometric features.

The work in driver's doziness is examined using measures like behavioural psychological and vehicular which makes this a hybrid doziness revelation system.

Shows revelation of doziness based on head movement and geometrical features of mouth is proffer .Experiment was conducted on sample size of 50 video clips and observed that head movement contributes about $8 \%$ and yawning contributes about $49 \%$. 


\section{III.PROPOSED SYSTEM}

The driver's face is constantly recorded using a video camera that is set-up under the front mirror. In order to detect the doziness, the first step is to analyse and track the face using the sequence of frame shots taken by the camera. Then the position of the eyes is recognized in the face. The closed eye gesture is identified along with closed eyes for dozy revelation. This makes section method more robust to false revelations.

The maw and eye geometrical features are then used to analyse the yawn. The system will warn the driver of his fatigue by use of an alarm or sound and the improper driving situation in case of doziness revelation.

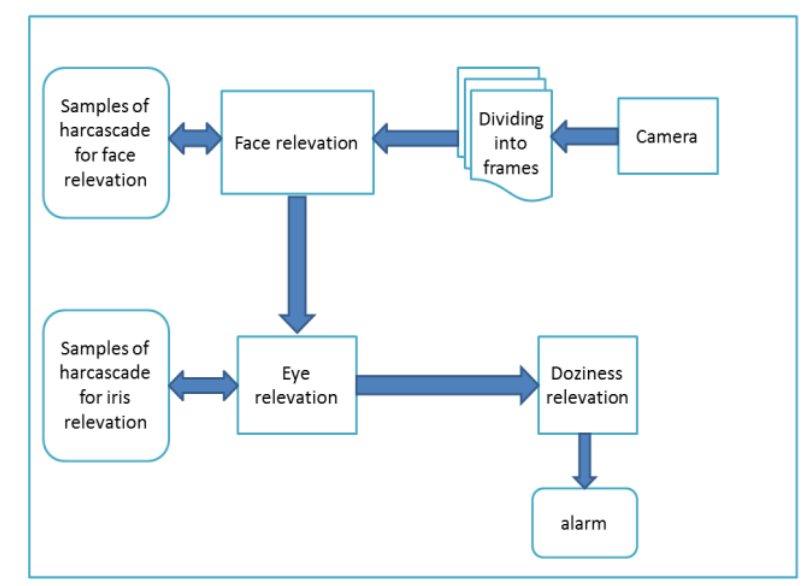

Figure 1 System Architecture

System architecture is the model that explains the construction behaviour, and more vision of a system.

An architecture narration is a formal description and illustration of a system, arrange in a way that supports reasoning about the construction and behaviours of the system.

A system architecture can contain system components, the broaden systems developed, that will work together to implement the complete system.

There have been endeavour to formalize languages to explain system architecture; commonly these are called architecture description languages (ADLs).

\section{MODULES:}

\section{Video acquisition:}

Video acquisition mainly implies attaining the live video feed of the automobile driver. Video acquisition is acquired, by making use of a camera.

\section{Dividing into frames:}

This part is used to take live video as its input and convert it into a sequence of frames/ images, which are then processed.

\section{Face revelation:}

The face revelation function takes one frame at a time from the frames provided by the frame capturer and in each and every frame it tries to analyse the face of the automobile driver. This is acquired by utilizing set of predefined Haarcascade samples.

\section{Eyes revelation:}

Once the face revelation function has analysed the face of the automobile driver, the eyes revelation function tries to analyse the automobile driver's eyes. This is acquired by utilizing set of pre-defined Haarcascade samples.

\section{Doziness revelation:}

After identifying the eyes of the automobile driver, the doziness revelation function analyse if the automobile driver is dozy or not, by examining the state of the eyes , that is, open or closed and the squint rate.

\section{IV.HAARCASCADECLASSIFIER ALGORITHM}

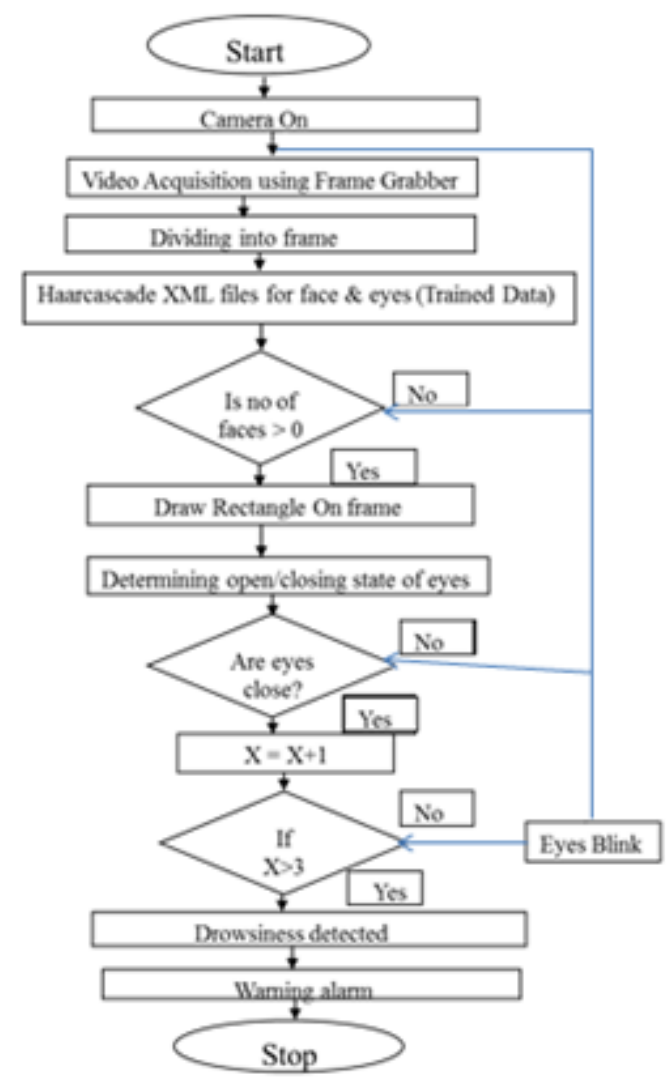

Figure 2: HAARCASCADE Flowchart

\section{HAAR CASCADE CLASSIFIERS}

The gist base for Haar classifier object revelation is the Haar-like features. Two or three abutting groups with a relative difference form a Haar-like feature.

These features, rather than using the potency values of a pixel, use the change in contrast values between abutting rectangular groups of pixels.

The contrast differences between the pixel groups are used to analyse relative light and dark areas.

Haar-like features are used to analyse an image.

Haar features can easily be calibrated by raising or reducing the size of the pixel group being analysed.

This allows features to be used to analyse objects of various sizes. 


\section{ADVANTAGES}

\section{Reduce Mishaps or Accidents:-}

As we are using this system, the accidents rate will probably decrease because if by chance driver sleeps or takes a nap an alert beep or buzzer will start; by which driver will be alert.

\section{Ensure Road Safety:-}

Road safety will be maintained.

\section{Hassel Free Driving}

\section{CONCUSION}

Doziness affects mental agility, reducing an individual's capability to perform a vehicle safely and raising the risk of human fault that could lead to disasters and injuries. In Addition, it has been shown to slow reaction time, reduces perception, and impairs judgment.

Long hours behind the wheeling soporific driving environments make truck drivers particularly prone to dozy - driving crashes successfully addressing the concern of driver doziness in the commercial motor vehicle industry is dreadful and multi-faceted challenge. Since a large number of road mishaps occur due to the driver doziness this system will be helpful in preventing many mishaps or accidents, and consequently save money and decrease personal suffering. This system will monitor the driver's eyes using a camera and by evolving an algorithm we can analyse symptoms of driver fatigue early enough to avoid an mishap.

So this project will be helpful in analysing driver fatigue in advance and will gave alerting output in form of sound. [3]

\section{REFERENCES}

[1] Drowsy Driver Warning System Using Image Processing

[2] 1 Singh Himani Parmar, 2 Mehul Jajal, 3 Yadav Priyanka Brijbhan INTERNATIONAL JOURNAL OF ENGINEERING DEVELOPMENT AND RESEARCH

[3] http://www.ijedr.org/papers/IJEDR1303017.pdf

[4] https://www.slideshare.net/ijtra/eye-tracking-based-driverdrowsiness-monitoring-and-warning-system 